\title{
Actividades de prevención y factores de riesgo en diabetes mellitus y pie diabético
}

\author{
Prevention activities and risk factors in diabetes \\ mellitus and diabetic foot
}

\author{
Análida Elizabeth Pinilla, María del Pilar Barrera, Cristhian Rubio, \\ Diego Devia • Bogotá, D.C. (Colombia)
}

\section{Resumen}

Objetivos: determinar prevalencia actividades de prevención por médicos y pacientes. Identificar factores de riesgo: pie, metabólicos, alimentarios y estado nutricional.

Métodos: estudio descriptivo, transversal octubre 2009-junio 2011, pacientes hospitalizados con diabetes mellitus, mayores de edad; aprobación comité ética. Encuesta médica, evaluación nutricional, medición A1c y lípidos, educación mediante cartilla autocuidado.

Análisis descriptivo: variables cuantitativas y cualitativas, coeficientes correlación de Pearson; programas Excel 2007 y SPSS-versión-15.0.

Resultados: pacientes encuestados 263; hombres $44.1 \%$ y mujeres $55.9 \%$; edad promedio 63.6 \pm 13.3 ; tiempo diagnóstico diabetes $11.4 \pm 8.5$ años. Escolaridad primaria $58.9 \%$, estrato socioeconómico 1-2 (71.3\%). Antecedente: úlceras 14.8\%, amputación 1.9\%, disestesias 49.8\%, claudicación intermitente $22.7 \%$, examen pies por médico $42.9 \%$.

Actividades prevención por médicos son precarias porque no: educan $59.5 \%$ sobre autocuidado pie, examinan pies $57.1 \%$, preguntan sobre: disestesias $68.3 \%$, claudicación intermitente $74.8 \%$. Autocuidado pacientes diario inadecuado porque, no revisan: pies $27.4 \%$, interior calzado $28.1 \%$; no realizan: secado interdigital $8 \%$, lubricación pies $58.8 \%$; sin automonitoreo $63.2 \%$; corte inadecuado uñas $68.1 \%$; no usan: medias $21.7 \%$, calzado terapéutico $98.5 \%$; sin colaboración familiar $55.1 \%$.

Descontrol metabólico: A1c 9.6\% \pm 2.7 ; c-LDL $99.1 \mathrm{mg} \% \pm 31.3$; triglicéridos: $171.9 \mathrm{mg} \% \pm 97.9$.

Sin consejería por nutricionista $39.4 \%$; con preobesidad-obesidad 58.9\%. Correlación: IMC y cintura punto medio $(\mathrm{r}=0.750, \mathrm{p}=0.000)$; IMC y porcentaje grasa corporal $(\mathrm{r}=0.586, \mathrm{p}=0.000)$. Hábitos alimentación: preferencia alimentos fritos $42.3 \%$; bajo consumo: proteína animal (12.7\%), lácteos $(31.8 \%)$, frutas $(64.7 \%)$ y verduras $(57.9 \%)$; alto consumo almidones $(43.4 \%)$.

Conclusiones: las actividades de prevención por médicos y pacientes son deficientes. Se evidenciaron factores de riesgo para diabetes mellitus y pie diabético: hiperlipidemia, hiperglucemia, obesidad y hábitos alimentarios inadecuados. (Acta Med Colomb 2014; 39: 250-257)

Palabras clave: diabetes mellitus, prevención, factores de riesgo, pie diabético, nutrición, educación.

\footnotetext{
Abstract

Objectives: to determine prevalence and prevention activities by physicians and patients. Identify risk factors: foot, metabolic, dietary and nutritional status.

Methods: a descriptive, cross-sectional study, from October 2009 to June 2011 in adult patients hospitalized with diabetes mellitus. Ethics Committee approval. Medical questionnaire, nutritional assessment, measurement A1c and lipids, education through self care booklet.

Descriptive analysis: quantitative and qualitative variables, Pearson correlation coefficients; Excel 2007 and SPSS version-15.0 programs.

Results: 263 patients were surveyed; $44.1 \%$ men and $55.9 \%$ women; mean age $63.6 \pm 13.3$; time of diabetes diagnosis $11.4 \pm 8.5$ years. $58.9 \%$ had primary schooling, socioeconomic status $1-2(71.3 \%)$.

Clinical antecedents: ulcers $14.8 \%, 1.9 \%$ amputation, dysesthesias $49.8 \%, 22.7 \%$ intermittent claudication, foot exam by physician $42.9 \%$.
}

Dra. Análida Elizabeth Pinilla Roa: Internista. PhD en Educación. Formación en Nutrición y Diabetes. MSc en Educación con Énfasis en Docencia Universitaria. Especialista en Evaluación y Construcción de Indicadores de Gestión para la Educación Superior. Profesora Asociada Facultad de Medicina, Universidad Nacional de Colombia; Dra. María del Pilar Barrera Perdomo: MSc. en Nutrición Clínica y en Administración Educativa. Profesora Titular, Departamento de Nutrición Humana, Facultad de Medicina, Universidad Nacional de Colombia; Dres. Cristhian Rubio Ramos y Diego Devia Manchola: Residentes de Medicina Interna. Bogotá, D.C. (Colombia).

Correspondencia. Análida Elizabeth Pinilla Roa. Bogotá, D.C. (Colombia).

E-mail: aepinillar@unal.edu.co Recibido: 18/VI/2013 Aceptado: 1/VIII/2014 
Prevention activities by doctors are precarious because $59.5 \%$ do not educate about foot self-care, $57.1 \%$ examine feet, $68.3 \%$ ask about dysesthesias, $74.8 \%$ about intermittent claudication. Inadequate daily feet self-care by patients because they don't inspect their feet $27.4 \%$ or the inner shoes $28.1 \%$; $8 \%$ do not make interdigital drying; $58.8 \%$ do not lubricate their feet; $63.2 \%$ make no self-monitoring; $68.1 \%$ have inadequate nail cutting; $21.7 \%$ do not use stockings, $98.5 \%$ do not have therapeutic footwear; $55.1 \%$ have no family collaboration.

Metabolic dyscontrol: A1c 9.6 $\pm 2.7 \%$; LDL-C 99.1\% $\pm 31.3 \mathrm{mg}$; triglycerides: $171.9 \pm 97.9 \mathrm{mg} \%$.

$39.4 \%$ do not have nutritionist counseling; $58.9 \%$ present pre-obesity-obesity. Correlation: BMI and waist midpoint circumference $(\mathrm{r}=0.750, \mathrm{p}=0.000)$; $\mathrm{BMI}$ and body fat percentage $(\mathrm{r}=0.586, \mathrm{p}=$ $0.000)$. Eating habits: fried food preference $42.3 \%$; Low consumption: animal protein $(12.7 \%)$, dairy $(31.8 \%)$, fruits $(64.7 \%)$ and vegetables $(57.9 \%)$; high starch consumption (43.4\%).

Conclusions: prevention efforts by doctors and patients are deficient. Risk factors for diabetes mellitus and diabetic foot observed were : hyperlipidemia, hyperglycemia, obesity and inadequate eating habits. (Acta Med Colomb 2014; 39: 250-257)

Keywords: diabetes mellitus, prevention, risk factors, diabetic foot, nutrition, education.

\section{Introducción}

La diabetes mellitus (DM) es un problema de salud pública, causa un impacto importante en la morbimortalidad, por las complicaciones crónicas que se relacionan con la carencia de intervenciones oportunas para identificar los factores de riesgo que inciden en el desarrollo de lesiones de órganos blanco (1).

La DM es la principal causa de amputación no traumática en miembros inferiores, generalmente es precedida de úlcera en el pie del paciente. Además, cerca de la mitad de los pacientes amputados por primera vez requerirán amputaciones adicionales en los siguientes tres años y la mitad de los pacientes a quienes se les practique una amputación mayor morirán en cinco años (2). Sin lugar a dudas, la identificación de los pacientes en riesgo es el primer paso para alcanzar este objetivo, seguido de la educación al paciente (3).

Es de mencionar, que médicos generales y especialistas se olvidan de realizar una evaluación integral del paciente para realizar un diagnóstico precoz de retinopatía, nefropatía, pie diabético en riesgo, entre otros; es por esto, que el médico internista tiene la función de hacer una evaluación de las diversas complicaciones de la DM y, asimismo, de los factores de riesgo y focalizar su esfuerzo hacia la prevención primaria en los diferentes escenarios de acción, ambulatorio u hospitalarios $(4,5)$. Igualmente, al hacer un tratamiento integral del paciente con DM, es necesario enfatizar en los cambios en el estilo de vida asociados con la alimentación y la nutrición, que son aspectos importantes para prevenir la lesión en el pie y demás órganos blanco (cerebrovascular, coronario, renal, etc). A pesar de todo, en Colombia, pocas investigaciones han indagado a profundidad la aplicación de estrategias de prevención para DM tipo 2 (DM2) y pie diabético.

Por lo anterior, se realizó esta investigación con el objetivo de determinar la prevalencia de actividades de prevención del pie diabético recomendadas por los médicos y efectuadas por los pacientes con DM hospitalizados en el servicio de medicina interna de la Clínica Universitaria Carlos Lleras Restrepo de Bogotá; además, caracterizar los factores de riesgo metabólicos relacionados como la hemoglobina glicosilada (A1c) y el perfil lipídico, y los asociados con la alimentación y el estado nutricional.

\section{Material y métodos}

Estudio descriptivo de corte transversal. La población estudio incluyó pacientes con DM, mayores de 18 años, hospitalizados en los servicios de medicina interna.

La muestra estuvo conformada por 263 pacientes con DM2 a quienes se encuestó y evaluó en forma consecutiva y que cumplían con los criterios de inclusión (mayores de 18 años, hospitalizados por primera vez, por más de tres días y que aceptaran participar en el estudio). Los criterios de exclusión fueron: paciente menor de 18 años, minimental menor de 20, DM diagnosticada por primera vez, presencia de edemas de miembros inferiores de cualquier grado o no asistir a control médico ambulatorio en el último año.

El protocolo de investigación fue aprobado por el Comité de Ética de la Facultad de Medicina, se consideraron las "Normas Científicas, Técnicas y Administrativas para la Investigación en Salud" según Resolución No. 008430 de 1993 del Ministerio de Salud.

La información se recolectó en el periodo comprendido entre octubre de 2009 y junio de 2011. Se aplicó una encuesta a los pacientes (historia clínica con el manual de definiciones), a cada paciente se le solicitó consentimiento informado. El cuestionario constaba de: datos generales de identificación del paciente con variables sociodemográficas, antecedentes de riesgo para pie diabético, actividades médicas de prevención en el último año, actividades de prevención y autocuidado realizadas por el paciente, metabólicas (A1c y perfil lipídico -colesterol total, c-LDL, cHDL y triglicéridos- según los criterios del Adult Treatment Panel III (6). Asimismo, se hizo la evaluación nutricional y dietética con: antropometría (peso, talla, índice de masa 
corporal -IMC-, perímetro de la cintura -PC-, perímetro del brazo, pliegue del triceps, perímetro de la pantorrilla), dinamometría, examen físico y hábitos alimentarios $(7,8)$. Al terminar cada encuesta, para educar al paciente, se entregó y explicó la "Guía de autocuidado del pie del diabético", que incluía recomendaciones generales para la DM, pie diabético y alimentación.

\section{Análisis estadístico}

La información recolectada se consignó en una base de datos en Excel Office 2007 y se procesó en el programa SPSS versión 15.0. Las variables estudiadas se analizaron con medidas de tipo descriptivo como proporciones para variables cualitativas y promedios o medias con sus respectivas medidas de variabilidad, en el caso de las variables cuantitativas. Se usaron tablas de contingencia para la exploración de posibles asociaciones entre variables. Además, se determinaron coeficientes de correlación de Pearson.

\section{Resultados}

Se incluyeron 263 pacientes para el análisis de medicina interna y 221 para el componente de nutrición. Del total de la muestra, el $55.9 \%(n=147)$ correspondió a mujeres y el $44.1 \%$ $(\mathrm{n}=116)$ a hombres; rango de edad entre 21 y 95 años, con promedio $63.6 \pm 13.3$ años. La mayor parte de los pacientes reportaron haber cursado la primaria (58.9\%), seguidos de los que cursaron secundaria (20.5\%), el $12.9 \%$ eran analfabetas. La mayor parte pertenecía al estrato 2 (57.4\%), seguidos del estrato 3 (26\%), estrato 1 el $14 \%$. El tiempo promedio del diagnóstico de la DM fue $11.4 \pm 8.5$ años.

\section{Variables de prevención}

Los pacientes informaron asistir a control para la DM2, así: $53.8 \%$ cada mes, $40 \%$ cada $2-3$ meses, $4.6 \%$ cada seis meses y $1.5 \%$ cada año; 36 pacientes reconocieron ser fumadores activos, sin embargo, sólo 21 (58\%) informaron que el médico les recomendó dejar de fumar. Como se observa en la Tabla 1, 39 pacientes (14.8\%) tenían antecedente de úlcera en pies y cinco pacientes (1.9\%), antecedente de amputación. De aquellos con antecedente de úlcera, 33 pertenecían a estratos socioeconómicos 1 y 2 , de los cuales 29 tenían escolaridad primaria y cinco eran analfabetas.

Además, 131 pacientes (49.8\%) tenían disestesias (alteración de la sensibilidad), pero a 179 (68.3\%) del total de los pacientes encuestados, el médico tratante en consulta externa no los había interrogado sobre este síntoma;

Tabla 1. Antecedentes de riesgo en la muestra total.

\begin{tabular}{|l|c|c|}
\hline Antecedente & $\mathbf{N}^{\circ}$ pacientes & $\%$ \\
\hline Úlcera en pies & 39 & 14.8 \\
Amputación & 5 & 1.9 \\
Disestesias & 131 & 49.8 \\
Claudicación intermitente & 59 & 22.7 \\
\hline
\end{tabular}

situación similar se encontró con claudicación intermitente, 59 pacientes $(22.7 \%$ ) afirmaron tenerla pero $74.8 \%$ del total de los pacientes encuestados, no habían sido interrogados al respecto (Tabla 1 ).

En general, las actividades de prevención para pie diabético realizadas por los médicos resultaron precarias, puesto que $59.5 \%$ de los pacientes informaron no haber recibido instrucciones sobre el autocuidado del pie, únicamente el 40.5\% ( $\mathrm{n}=106)$ afirmó haber recibido instrucciones para el autocuidado de los pies; asimismo, $57.1 \%$ de los pacientes informaron que el médico de consulta externa no les había examinado los pies, es decir, en el último año sólo al $42.9 \%$ de los pacientes les habían revisado los pies; de igual forma, tampoco les habían preguntado sobre disestesias al 68.3\% y claudicación intermitente al $74.8 \%$ del grupo total de los pacientes encuestados.

En relación con las recomendaciones adecuadas por parte de los médicos, las más frecuentes fueron: no caminar descalzo $(74.5 \%)$, usar calzado terapéutico para proteger pies $(69.8 \%)$, uso diario de medias $(59.4 \%)$ y revisión diaria de los pies $(55.7 \%)$.

Las actividades adecuadas más frecuentes efectuadas por los pacientes para el autocuidado y prevención del pie diabético fueron: secado de los espacios interdigitales después del baño todos los días $92 \%$, no caminar descalzo $90.9 \%$, tener en cuenta la temperatura del agua a la hora de bañar los pies $73.4 \%$, uso de medias todos los días $63.1 \%$, revisión del interior del calzado antes de introducir el pie $65 \%$, revisión diaria de los pies $57.4 \%$. En contraste, las actividades inadecuadas más frecuentemente realizadas por los pacientes fueron: no tener en cuenta el color de las medias que usan $92.4 \%$, no usar calzado terapéutico que proteja los pies $98.5 \%$, corte inadecuado de las uñas $68.1 \%$, no realizar automonitoreo de la glucemia $63.2 \%$, no lubricar los pies todos los días $58.8 \%$, no recibir colaboración de familiar para cuidar los pies $55.1 \%$.

En la Tabla 2 se comparan las actividades que los pacientes informaron realizar versus las que los médicos les habían recomendado. Uno de los objetivos del estudio era determinar si las medidas que el médico recomendaba se veían reflejadas en su aplicación por parte de los pacientes; al analizar los datos comparativos se observa que aunque se recomienda el uso de calzado terapéutico, sólo lo usa un porcentaje de pacientes bajo, $0.8 \%$.

\section{Variables metabólicas (pruebas de laboratorio)}

$\mathrm{Al} 82.9 \%$ se le midió A1c, con promedio $9.6 \% \pm 2.7$, con valor en meta, menor o igual a 7\%, en $20.2 \%$ de las muestras analizadas. En cuanto al perfil lipídico estaban en metas: colesterol total $73.4 \%$ (meta: $<200 \mathrm{mg} / \mathrm{dL}$ ), triglicéridos 48.3\% (meta: $<150 \mathrm{mg} / \mathrm{dL}$ ), LDL $47.9 \%$ (meta: $<100 \mathrm{mg} /$ dL), c-HDL $20.1 \%$ (meta: > $40 \mathrm{mg} / \mathrm{dL}$ en hombres y $>50 \mathrm{mg} /$ dL en mujeres). Los rangos de estas variables metabólicas se observan en la Tabla 3, sobresale el promedio alto para cifras de A1c y triglicéridos. 
Tabla 2. Actividades de prevención realizadas por pacientes en comparación con las recomendadas por médicos.

\begin{tabular}{|c|c|c|}
\hline & $\begin{array}{l}\text { Actividades } \\
\text { realizadas } \\
\text { por pacientes } \\
(\mathbf{n}=\mathbf{2 6 3}) \\
\mathbf{1 0 0 \%}\end{array}$ & $\begin{array}{c}\text { Pacientes } \\
\text { instruidos por } \\
\text { los médicos } \\
(n=106) \\
\text { 40.5\% }\end{array}$ \\
\hline Revisión diaria de los pies & 57.4 & 55.7 \\
\hline Uso diario de medias & 63.1 & 59.4 \\
\hline Tener en cuenta el color de medias por comprar & 6.8 & 12.3 \\
\hline Uso de calzado terapéutico para proteger pies & 0.8 & 69.8 \\
\hline Revisión del interior del calzado al calzarse & 65 & 33 \\
\hline Uso diario de lubricantes en los pies & 32.1 & 49.1 \\
\hline Secado interdigital después del baño & 92 & 48.1 \\
\hline Temperatura del agua para bañar los pies & 73.4 & 10.4 \\
\hline Colaboración de familia para revisión de pies & 41.8 & 11.3 \\
\hline Realizar automonitoreo de glucemia & 32.6 & 34 \\
\hline No usar objetos calientes para calentar pies & 88.6 & 15.5 \\
\hline No utilizar callicidas & 95 & 14.2 \\
\hline No caminar descalzo & 90.9 & 74.5 \\
\hline Corte adecuado de las uñas & 30.4 & 36.8 \\
\hline
\end{tabular}

Tabla 3. Variables metabólicas.

\begin{tabular}{|l|c|c|c|c|c|}
\hline & $\mathbf{N}^{\circ}$ & Mínimo & Máximo & Media & D.E. \\
\hline Alc (\%) & 205 & 6.0 & 18.1 & 9.6 & 2.7 \\
Colesterol total (mg\%) & 244 & 27.5 & 330 & 163.7 & 43.1 \\
Triglicéridos (mg\%) & 244 & 38 & 712 & 171.9 & 97.9 \\
c-LDL (mg\%) & 240 & 50.0 & 192.8 & 99.1 & 31.3 \\
c-HDL (mg\%) & 244 & 12 & 90.0 & 35.2 & 11.9 \\
\hline
\end{tabular}

\section{Variables de nutrición}

El 36.7\% de los pacientes no recibió recomendaciones sobre alimentación por parte del médico y $39.4 \%$ no obtuvo consejería por un nutricionista dietista.

Con respecto al diagnóstico nutricional, 58.9\% $(\mathrm{n}=130)$, presentó malnutrición por exceso (preobesidad y obesidad) (Figura 1), con predominio en mujeres. En la Tabla 4 se presenta la descripción de las variables relacionadas con el estado nutricional.

La obesidad abdominal en punto medio fue de $77.3 \%$ para hombres y de $95.9 \%$ para mujeres, según parámetros de la International of Diabetes Federation (IDF), acogidos para Colombia por el Consenso Colombiano de Síndrome Metabólico ( $90 \mathrm{~cm}$ para hombres y $80 \mathrm{~cm}$ para mujeres); la obesidad abdominal medida en cresta ilíaca fue de $40 \%$ para hombres y de $91 \%$ para mujeres, según el ATP III (102 $\mathrm{cm}$ para hombres y $88 \mathrm{~cm}$ para mujeres) (6).

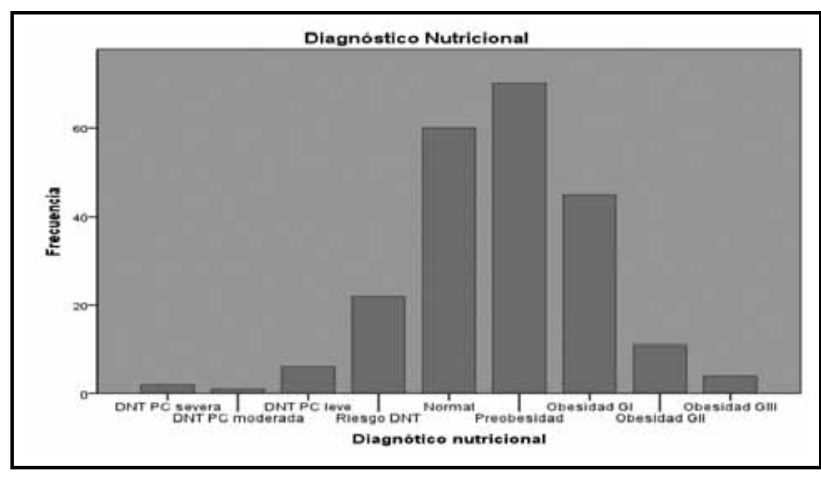

Figura 1. Diagnóstico nutricional.

Tabla 4. Descripción de variables del estado nutricional.

\begin{tabular}{|l|c|c|}
\hline Parámetro/Indicador & Media & D.E. \\
\hline Peso actual $(\mathrm{Kg})$ & 68.1 & 13.8 \\
Talla (cm) & 156.6 & 18.1 \\
IMC $\left(\mathrm{Kg} / \mathrm{m}^{2}\right)$ & 27.2 & 4.9 \\
Perímetro del brazo (cm) & 30.2 & 4.3 \\
CMB (cm) & 25.2 & 3.4 \\
Porcentaje de grasa corporal por sumatoria & 25.7 & 7.0 \\
de pliegues cutáneos & 99.8 & 12.1 \\
Perímetro de la cintura (cresta ilíaca cm) & 97.8 & 12.1 \\
Perímetro de la cintura (punto medio cm) & & \\
Perímetro de la pantorrilla (cm) & 33.4 & 3.0 \\
(En mayores de 65 años) & 21.8 & 11.6 \\
Fuerza muscular (Kg) & 76.2 & 2.5 \\
Fuerza muscular (\%) & & \\
\hline
\end{tabular}

En relación con la dinamometría, la media del porcentaje de fuerza muscular fue de $76.2 \% \pm 22.5$; en menores de 65 años correspondió a $77.6 \% \pm 22.6$ y en mayores de 65 años a $75.2 \% \pm 22.4$.

Se encontraron asociaciones importantes entre las variables antropométricas que indican adiposidad; además, se halló asociación positiva entre la circunferencia muscular del brazo (CMB) y la fuerza muscular. En la Tabla 5 y en las Figuras 2 y 3 , se observan las correlaciones más importantes. Es de mencionar en cuanto al sitio de medición de la cintura, que tanto el punto medio como la cresta ilíaca son representativos de la cantidad de tejido adiposo a nivel abdominal. Por otra parte, se aprecia que el IMC también tiene una relación importante con la distribución de grasa regional medida en la cresta ilíaca

En cuanto a los hábitos de alimentación, 33\% de los pacientes consumía tres comidas al día, seguido de $31.2 \%$ con cinco y de $18.6 \%$ con seis tiempos de comida; $3.2 \%$ consumía menos de dos comidas al día. En la Tabla 6, se 
Tabla 5. Asociaciones entre variables de estado nutricional.

\begin{tabular}{|c|c|c|}
\hline Variables & $\mathbf{r}$ & $\mathbf{p}$ \\
\hline Perímetro de la cintura en punto medio y perímetro de la cintura en cresta ilíaca & $0.84 * *$ & 0.00 \\
\hline IMC y perímetro de la cintura en cresta ilíaca & $0.84 * *$ & 0.00 \\
\hline IMC y perímetro de la cintura en punto medio & $0.75^{* *}$ & 0.00 \\
\hline IMC y\% grasa corporal sumatoria de pliegues & $0.59 * *$ & 0.00 \\
\hline Perímetro de la cintura en cresta ilíaca y\% grasa corporal sumatoria de pliegues & $0.52 * *$ & 0.003 \\
\hline Perímetro de la cintura en cresta ilíaca y\% grasa corporal sumatoria de pliegues & $0.52 * *$ & 0.00 \\
\hline Perímetro de la cintura en punto medio y $\%$ grasa corporal sumatoria de pliegues & $0.33 * *$ & 0.00 \\
\hline CMB y fuerza muscular $(\mathrm{Kg})$ & $0.25 * *$ & 0.00 \\
\hline
\end{tabular}

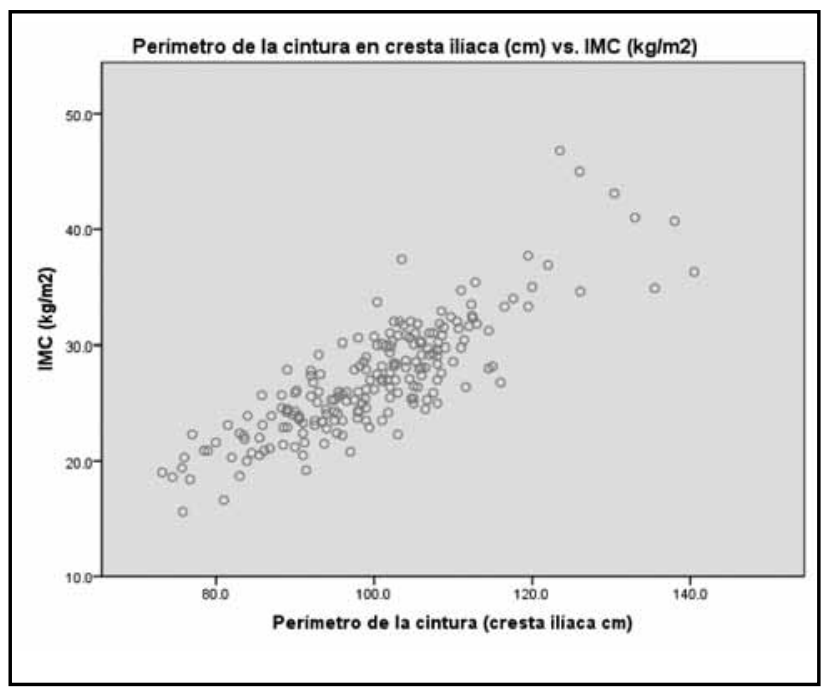

Figura 2. Correlación entre perímetros de cintura según lugar de medida.

Tabla 6. Hábitos de alimentación.

\begin{tabular}{|l|c|c|}
\hline Características de la alimentación & $\begin{array}{c}\mathbf{S i} \\
(\mathbf{\%})\end{array}$ & $\begin{array}{c}\text { No } \\
(\boldsymbol{\%})\end{array}$ \\
\hline Consumo de alimentos fritos & 42.3 & 57.7 \\
Consume carne, pescado, pollo o huevo diariamente & 87.3 & 12.7 \\
Consume productos lácteos mínimo dos veces al día & 68.2 & 31.8 \\
$\begin{array}{l}\text { Consume más de dos harinas al desayuno, } \\
\text { al almuerzo o a la comida }\end{array}$ & 43.4 & 56.6 \\
$\begin{array}{l}\text { Consume al menos cuatro porciones de frutas al día } \\
\text { Consume al menos dos porciones de verduras al día }\end{array}$ & 35.3 & 64.7 \\
$\begin{array}{l}\text { Adiciona azúcar, panela o miel a las preparaciones o } \\
\text { consume alimentos que lo contengan }\end{array}$ & 35.3 & 64.7 \\
Acostumbra utilizar el salero de mesa & 18.8 & 81.2 \\
\hline
\end{tabular}

presentan los resultados de las preguntas relacionadas con la alimentación. Se destaca: consumo diario de alimentos fuente de proteína de alto valor biológico, consumo bajo de lácteos, ingesta alta de carbohidratos con más de dos porciones en alguno de los tiempos principales de comida;

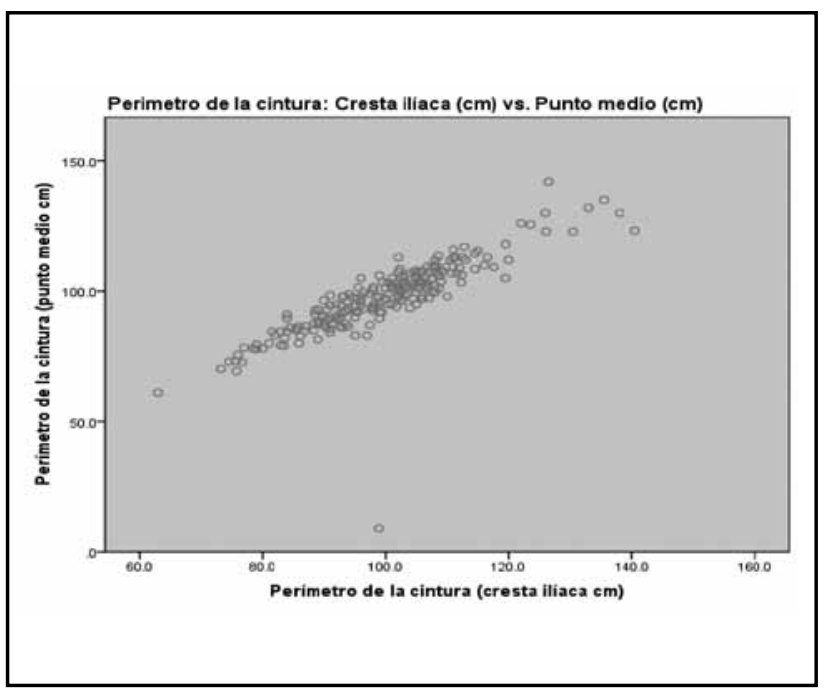

Figura 3. Correlación entre IMC y perímetro de la cintura en cresta ilíaca.

bajo aporte de verduras y frutas; utilización de azúcar, panela o miel de abejas en preparaciones.

\section{Discusión}

La mayor parte de los pacientes encuestados eran mayores de 65 años y correspondían al género femenino, lo cual coincide con los datos de los Indicadores Básicos de Salud en Colombia 2010, en los cuales la DM fue la cuarta causa de mortalidad en mujeres mayores de 45 años (9).

En este grupo de pacientes se encontró déficit en las actividades de prevención recomendadas por los médicos en el control ambulatorio y en las actividades que los pacientes reconocen realizar de forma cotidiana. En otro estudio, Pinilla et al, reportaron que los médicos de primer nivel de atención no habían realizado educación sobre el cuidado del pie al $78.2 \%$ de pacientes, de igual forma, no les habían examinado los pies al $76.2 \%$ en el último año, y a pesar de presentar disestesias y claudicación intermitente, no se había indagado sobre estos síntomas al 89 y $93 \%$ de pacientes respectivamente (4). Esta realidad colombiana contrasta con los lineamientos del Grupo Internacional de 
Pie Diabético que pertenece a la IDF, los cuales han insistido en el costo-efectividad de las actividades de prevención para reducir la prevalencia de úlceras y amputación (3). Se ha evidenciado que pacientes de alto riesgo para lesiones del pie diabético requieren de un diagnóstico oportuno y educación para apropiar conductas de autocuidado por la diabetes y de autoexamen de los pies (10-13).

Por lo anterior, todo clínico competente que brinde atención a una persona con DM ha de realizar el interrogatorio sobre factores de riesgo para pie diabético (disestesias, claudicación intermitente, úlcera y amputación), el examen fundamental del pie (inspección, sensibilidad protectora con el monofilamento de Semmes Weinstein, la vibración con el diapasón de $128 \mathrm{~Hz}$ ) y la exploración para enfermedad vascular periférica, con la palpación comparativa de pulsos arteriales de los miembros inferiores y si están alterados cuantificar el índice tobillo/brazo (relación de la presión arterial sistólica) $(3,4,14,15)$.

Es claro que la visión del médico internista es integral, es decir, que al trabajar sobre aspectos nutricionales, metabólicos como la HbA1c y los lípidos se están previniendo todas las lesiones de órganos blanco y, frente al paciente, es útil trabajar sobre educación en pie, como estrategia didáctica, puesto que un paciente puede detectar las lesiones dermatológicas y comprender que al prevenir las lesiones de los pies está previniendo otras lesiones sistémicas.

Con este estudio se evidencia que la identificación de la neuropatía diabética y de la enfermedad arterial periférica por medio de la anamnesis y el examen físico y las recomendaciones de cuidado, continúan siendo actividades preventivas subutilizadas para disminuir el riesgo de aparición de úlceras del pie, lo cual puede terminar en amputación menor si está por debajo de tobillo o mayor si es a nivel de pierna o muslo $(10,11)$. Lo anterior acarrea deterioro funcional, psíquico y económico, convirtiéndose por tanto en la discapacidad más frecuente de los pacientes con DM2.

Por esto, es necesario repensar la formación médica en temas relacionados con la DM2 y las diferentes complicaciones de esta enfermedad, como pie diabético, que suele tener complicaciones devastadoras: úlcera, amputación y muerte. Tanto esta investigación con pacientes hospitalizados como una anterior con pacientes ambulatorios revelaron que una proporción importante de médicos no interroga sobre disestesias y claudicación intermitente en lo que utilizaría sólo unos pocos segundos. En otras palabras, se continúa con una formación profesional basada en el conocimiento "memorístico", pero falta su aplicación eficiente y, además, la actitud de brindar servicio y cuidado al paciente; por tanto, se debe virar hacia un desarrollo de competencias profesionales que integren el saber, el hacer y el saber ser (conocimientos, habilidades y actitudes) $(4,16)$.

La identificación de los pacientes en riesgo es el primer paso para alcanzar este objetivo, por lo tanto es importante indagar sobre antecedentes de disestesias, claudicación intermitente, úlcera y amputación. Se concluye que es fun- damental que cada paciente y su familia reciban instrucciones básicas de autocuidado; lo más pertinente es organizar programas educativos que permitan un seguimiento para que el paciente mediante un proceso continuo, se apropie de las actividades de autocuidado diario general en DM2, como el automonitoreo de la glucemia y, en particular, de las actividades para el cuidado de los pies (3, 10-12).

Es urgente, virar de la atención terciaria hacia la prevención primaria en DM2 $(3,13)$; en lo referente al pie diabético, la prevención primaria consiste en medidas sencillas de cuidado diario, como el aseo e higiene podiátrica, apoyado por un familiar o el cuidador; el uso de calzado protector, el cuidado de patologías no ulcerativas como callos, xerosis, tiña pedis; además, todo médico debe realizar la clasificación del riesgo del pie si hay neuropatía, vasculopatía o antecedente de úlcera o amputación $(12,14,15)$.

En síntesis, implementar programas y actividades de educación previene complicaciones de la DM como: amputaciones, enfermedad coronaria y vascular cerebral, nefropatía diabética y retinopatía diabética. Por lo anterior, todo el equipo de salud tiene la responsabilidad de hacer prevención en todos los niveles de atención, para frenar el síndrome metabólico, la prediabetes y el desarrollo hacia la DM y por tanto, las lesiones crónicas secundarias a ésta.

Asimismo, se encontró un descontrol importante de las variables metabólicas. En general, la mayoría (79.8\%) se encontraba con A1c fuera de la meta planteada en esta investigación (7\%); sin embargo, actualmente este valor ha cambiado según las particularidades de cada paciente como la edad, el tiempo de evolución de la diabetes y las comorbilidades $(1,17,18)$. Llama la atención que sólo $32 \%$ de los pacientes informaron hacer automonitoreo de glucometría; sin embargo, es necesario aclarar que esta investigación se hizo en un periodo anterior al Acuerdo 029 de 2011 del Plan Obligatorio de Salud (POS), por medio del cual se autoriza la prescripción del glucómetro para pacientes con DM2 que utilicen insulina, es claro que las guías clínicas han insistido en la educación y el automonitoreo para lograr y mantener las metas del control de la glucemia con el fin de frenar la progresión de las complicaciones (1, 3, 17-19).

En cuanto a las variables de nutrición, el hecho de que un grupo importante de pacientes no recibiera consejería por parte de un nutricionista dietista, demuestra la carencia en términos de asesoría nutricional y refleja el desconocimiento de los médicos sobre la necesidad de la remisión a la consulta de nutrición, aspecto que refleja la falta de conciencia respecto a la relevancia del trabajo interdisciplinario que permite brindar una atención integral al paciente adulto con DM.

La alta prevalencia de preobesidad y de obesidad en los individuos estudiados, demuestra su asociación con la presencia de DM2. Lo anterior es un reflejo del panorama nacional, teniendo en cuenta que las Encuestas Nacionales de la Situación Nutricional en Colombia (ENSIN) 2005 y 2010 encontraron una alta prevalencia de preobesidad y obesidad. La ENSIN 2010, halló que el problema se ha incrementado, 
el sobrepeso o preobesidad fue de $34.6 \%$ y la obesidad de $16.5 \%$, situación que fue más prevalente en mujeres (20-22).

Es de mencionar que en el grupo estudiado, se midió la cintura en la cresta ilíaca, con los puntos de corte del ATP III y en el punto medio, recomendado por la OMS, con los valores propuestos por la IDF acogidos para Colombia; se encontró una mayor prevalencia de obesidad abdominal según criterios de la IDF; sin embargo, ambas mediciones mostraron una correlación importante $(6,23-26)$. La asociación significativa entre el IMC y PC indica la importancia de medir este perímetro en las consultas médica y nutricional, como una estrategia de tamizaje que permita instaurar actividades de prevención para disminuir factores de riesgo cardiometabólicos. Es evidente que la medida del perímetro abdominal se relaciona de forma estrecha con la grasa visceral, la cual es metabólicamente deletérea al producir factores inflamatorios.

En la actualidad se están revisando los puntos de corte del PC, la Asociación Latinoamericana de Diabetes (ALAD) en 2010, propuso que para Latinoamérica se acogieran los valores de $94 \mathrm{~cm}$ en hombres y de $88 \mathrm{~cm}$ en mujeres. Aschner et al., en 2011, recomendaron $94 \mathrm{~cm}$ para hombres y a $90 \mathrm{~cm}$ para mujeres, cifras que se relacionan con la grasa visceral medida por tomografía (27-29). Gallo et al.,(2013) realizaron una investigación en Medellín (Colombia), encontraron que los puntos de corte de PC que se asociaron con resistencia a la insulina mediante HOMA fueron de $92 \mathrm{~cm}$ para hombres y 88 para mujeres; sin embargo, es necesario aclarar que el PC se medió en "el sitio más estrecho entre el reborde costal y la cresta ilíaca" (p. 120) (30). El debate para concertar los puntos de corte sigue abierto, en Colombia siguen siendo vigentes los puntos de corte propuestos por la IDF: $90 \mathrm{~cm}$ para hombres y $80 \mathrm{~cm}$ para mujeres, así como, la medición del PC según la recomendación de la OMS que es en el punto medio entre el borde inferior de la última costilla y el borde superior de la cresta ilíaca $(25,31)$.

Por otra parte, la mayor parte de los individuos presentó menor fuerza de agarre de la mano, aspecto que se puede relacionar con el hecho de que la mayor parte eran adultos mayores y con la adinamia por patologías de base. Es importante señalar la asociación positiva que se encontró entre mediciones antropométricas como la $(\mathrm{CMB})$ y la fuerza muscular (22).

En cuanto a los hábitos alimentarios, un alto porcentaje de individuos tenía bajo número de tiempos de comida, con respecto a las recomendaciones en una persona con DM, además refirieron el hábito de consumir alimentos fritos, factor que se puede relacionar con el predominio de preobesidad y obesidad. Si bien es cierto, una alta proporción de pacientes refirió consumo diario de alimentos fuentes de proteína de origen animal (carnes y lácteos), el instrumento de anamnesis utilizado no está diseñado para cuantificar la ingesta de este nutriente, por lo cual no es posible asegurar que el consumo de estos alimentos cubriera el requerimiento proteico de los individuos estudiados. Además, se observó una ingesta alta de carbohidratos, lo cual se puede relacionar con el exceso de peso y con el control metabólico inadecuado (valores altos de A1c). El bajo consumo de verduras y de frutas en la mayoría de individuos disminuye el aporte de vitaminas antioxidantes, minerales y fibra. Además el uso de azúcar, panela o miel de abejas se puede relacionar con el exceso de peso y el inadecuado control metabólico (cifras altas de A1c y triglicéridos).

La consejería nutricional se orienta a evitar episodios de hipoglucemia e hiperglucemia y a disminuir las complicaciones. El objetivo es proporcionar al individuo la información necesaria para que se concientice de su autocuidado. Se requiere la educación individualizada desde el diagnóstico y durante el tratamiento y el reforzamiento con actividades grupales para modificar comportamientos y actitudes.

En Colombia en el año 2009, se publicó la Ley 1355 "por medio de la cual se define la obesidad y las enfermedades crónicas no transmisibles asociadas a ésta como una prioridad de salud pública y se adoptan medidas para su control, atención y prevención". Esta Ley busca promover la alimentación saludable, y el ejercicio físico y establece el 24 de septiembre como el Día Nacional de la Lucha contra la Obesidad y el Sobrepeso y su semana correspondiente, como la semana de hábitos de vida saludable (32).

Por tanto, es importante que todos los miembros del equipo de salud comprendan la importancia de instruir y educar al paciente sobre diferentes aspectos como la reducción de peso, el control de la hiperglucemia, el control de la dislipidemia y la hipertensión arterial, sin olvidar el hábito del ejercicio y la suspensión del tabaquismo; en este sentido, mediante la Ley 1335 de 2009 se expidieron algunas "disposiciones por medio de las cuales se previenen daños a la salud de los menores de edad, la población no fumadora y se estipulan políticas públicas para la prevención del consumo del tabaco y el abandono de la dependencia del tabaco del fumador y sus derivados en la población colombiana" (33). De ahí el hecho de la importancia del trabajo interdisciplinario, en los diferentes niveles de atención, en los cuales hay que enfatizar en la prevención primaria de todos los factores de riesgo mencionados.

\section{Conclusiones}

A pesar de que las actividades de prevención tienen evidencia que respalda su implementación para modificar la historia natural de la DM y el pie diabético, las actividades de prevención realizadas por los médicos y efectuadas por los pacientes continúan siendo deficientes.

Se encontró un predominio de preobesidad y obesidad asociado con hábitos alimentarios que podrían favorecer la aparición de complicaciones de la DM y control metabólico inadecuado (cifras altas de A1c y triglicéridos).

\section{Agradecimientos}

A la División de Investigación Sede Bogotá de la Universidad Nacional de Colombia, Código DIB 10476, por el apoyo financiero y a las Directivas de la Clínica Universitaria Carlos Lleras Restrepo. A las estudiantes de Nutrición y Dietética: Lida Marcela 
Caicedo, Yuri Milena Castillo, Yani María Lozano y Karen Marcela Rodríguez; a la médica general Dra. Francy Saavedra.

\section{Fuentes de financiación}

La investigación fue financiada por la División de Investigación de la Sede Bogotá. Universidad Nacional de Colombia, código 10476.

\section{Referencias}

1. Diabetes Association. Standards of Medical Care in Diabetes. Diabetes Care 2013; 35(Suppl.1): S 11-63.

2. Levin and $\mathrm{O} \notin$ Neal $\phi$ s The diabetic foot. In: Bowker JH, Pfeifer MA, editors. Seventh Edition. Philadelphia: Mosby Elsevier; 2008.

3. International Consensus on the Diabetic Foot \& Practical Guidelines on the Management and Prevention of the Diabetic Foot. International Working Group on the Diabetic Foot (IWGDF). 2011.

4. Pinilla AE, Sánchez AL, Mejía A, Barrera MP. Actividades de prevención del pie Diabético en pacientes de consulta externa de primer nivel. Rev Salud Pública 2011; 13(2): 262-273.

5. Pinilla AE. Medicina Interna y Perfil del Internista. En: Archila PE, Senior JM Editores. Texto de Medicina Interna. Aprendizaje basado en problemas. Bogotá: Editorial Distribuna Editorial. p. 7-22.

6. Expert Panel on Detection, Evaluation, and Treatment of high Blood Cholesterol in Adults. Executive Summary of the Third Report of the National Cholesterol Education Program (NCEP) Expert Panel on Detection, Evaluation, and Treatment of High Blood Cholesterol in Adults (Adult Treatment Panel III). JAMA 2001; 285: 2486-97.

7. Ángel LA, Barrera MP. Evaluación nutricional de adulto hospitalizado. En: Murgueitio R, Prada GD, Archila PE, Pinzón A, Pinilla AE y col. Editores. Asociación Colombiana de Medicina Interna. Métodos diagnósticos en medicina clínica. Enfoque práctico. Bogotá: Celsus; 2007. p.163-72.

8. Lohman T, Roche A, Martorell R. Anthropometric standarization referent manual. Human Kinetics Books, Illinois, 1988.

9. Organización Panamericana de la Salud, Ministerio de la Protección Social República de Colombia, Instituto Nacional de Salud. Indicadores Básicos. Situación de Salud en Colombia. 2010.

10. De Berardis G, Pellegrini F, Franciosi M, Belfiglio M, Di Nardo B, Greenfield S, et al. Physician attitudes toward foot care education and foot examination and their correlation with patient practice. Diabetes Care 2004; 27: 286-7.

11. De Berardis G, Pellegrini F, Franciosi M, Belfiglio M, Di Nardo B, Greenfield S, et al. Are Type 2 diabetic patients offered adequate foot care? The role of physician and patient characteristics. J Diabetes Complications 2005; 19: 319-27.

12. Pinilla AE, Barrera MP. Manual para la prevención de la diabetes mellitus y complicaciones como el pie diabético. Segunda edición. Bogotá: Editorial Universidad Nacional de Colombia; 2013. ISBN 978-958-861.449-7.

13. Lavery LA, Wunderlich RP, Tredwell JL. Disease management for the diabetic foot: effectiveness of a diabetic foot prevention program to reduce amputations and hospitalizations. Diabetes Res Clin Pract 2005; 70: 31-7.

14. Pinilla AE. Pie diabético. En: Murgueitio R, Prada GD, Archila PE, Pinzón A, Pinilla AE, Londoño N y colaboradores. Métodos diagnósticos en Medicina Clínica. Primera edición. Bogotá: Editorial Médica Celsus; 2006. p. 511-523.

15. Márquez G, Pinilla AE. Pie Diabético. En: Archila PE, Senior JM Editores. Texto de Medicina Interna. Aprendizaje basado en problemas. Bogotá: Editorial Distribuna Médica: 2012. p. 1331-42.

16. Pinilla, A.E. Aproximación conceptual a las competencias profesionales en ciencias de la salud. Revista de Salud Pública 2012; 14: 852-64.
17. Acuerdo Número 29 de 2011. Que define, aclara y actualiza el Plan Obligatorio de Salud. Citado el 8 de junio de 2013. Disponible en: http://www.cres.gov.co/ Portals/0/acuerdo29de2011.pdfAmerican

18. AACE Comprehensive Diabetes Management Algorithm. Endoc Pract 2013; 19(2): 327-36.

19. Pinilla AE, Lancheros L, Viasus DF. Guía de atención de la diabetes mellitus tipo 2. En: Guías de promoción de la salud y prevención de enfermedades en la salud pública. Bogotá; Ministerio de la Protección Social de Colombia; 2007. p. 361-439.

20. Instituto Colombiano de Bienestar Familiar ICBF. Profamilia, Instituto Nacional de Salud, Universidad de Antioquia. Encuesta Nacional de la Situación Nutricional en Colombia. 2005. Panamericana Formas e Impresos S.A. Primera edición. Bogotá. 2006.

21. Ministerio de la Protección Social. Instituto Nacional de Salud, Instituto colombiano de Bienestar Familiar ICBF, Profamilia. Resumen Ejecutivo ENSIN 2010. Bogotá. 2011.

22. Barrera MP, Pinilla AE, Caicedo LM, Castillo YM, Lozano YM, Rodríguez KM. Factores de riesgo alimentarios y nutricionales en adultos con diabetes mellitus. Revista de la Facultad de Medicina Universidad Nacional 2012; 60(Supl 1): 308-20.

23. Asociación Colombiana de Endocrinología. Autores varios. (Barrera MP: Miembro del Jurado). Consenso Colombiano de Síndrome Metabólico. Bogotá; 2006.

24. Zimmet P. Alberti KG. Serrano M. Una nueva definición mundial de síndrome metabólico propuesta por la Federación Internacional de Diabetes: fundamento y resultados. Rev Esp Cardiol 2005; 58: 1371-6.

25. Wang J, Thornton J, Bari S, Williamson B, Gallagher D, Heysmsfield S, et al. Comparisons of waist circumferences measured at 4 sites. Am J Clin Nutr 2003; 77: 379-84.

26. Barrera MP, Pinilla AE, Cortés E, Mora G, Rodríguez María N. Síndrome metabólico: una mirada interdisciplinaria. Rev Colomb Cardiol 2008; 15 (3): 111-26.

27. Rosas-Guzmán J, Lyra R. Documento de posición de ALAD con aval de sociedades de diabetes y endocrinología latinoamericanas para el tratamiento de la diabetes tipo 2. Rev Endocrinol Nutr 2010; 18: 108-19.

28. Rosas J, González A, Aschner P, Bastarrachea R, editores. Asociación Latinoamericana de Diabetes (ALAD). Consenso Latinoamericano. Epidemiología, diagnóstico, control, prevención y tratamiento del síndrome metabólico en adultos. ALAD 2010; 18(1): 25-44.

29. Aschner P, Buendía R, Brajkvich I, González A, Figueredo R, Juárez XE, et al. Determination of the cutoff point for waist circumference that establishes the presence of abdominal obesity in Latin American men and women. Diab Res Clin Pract 2011; 93: 243-7.

30. Gallo JA, Ochoa J, Valparda JK, Aristizabal D. Puntos de corte del perímetro de la cintura para identificar sujetos con resistencia a la insulina en una población colombiana. Acta Med Colomb 2013; 38: 118-26.

31. Fundación Colombiana de Obesidad (Funcobes). Guía colombiana para el manejo científico de la obesidad. Disponible en: http://www.funcobes.org/ documentos.html\#ancla_guia. Consultado el 8 de diciembre de 2013.

32. República de Colombia. Ley de Obesidad 1355, por la cual se define la obesidad y las enfermedades crónicas asociadas a ésta como una prioridad de salud pública y se adoptan medidas para su control, atención y prevención. Diario oficial 14 octubre 2009.

33. República de Colombia. Ley 1335, por medio de las cuales se previenen daños a la salud de los menores de edad, la población no fumadora y se estipulan políticas públicas para la prevención del consumo del tabaco y el abandono de la dependencia del tabaco del fumador y sus derivados en la población colombiana. Diario Oficial 21 de julio de 2009. 